\title{
动力学拆分合成新型的基于氧化吲哚的轴手性苯乙烯化合物
}

\author{
江梦雪 $a$ 周涛*,b史炳锋 $*, b$ \\ ( ${ }^{a}$ 五邑大学化学与环境工程学院 广东江门 529020) \\ ( ${ }^{b}$ 浙江大学化学系 杭州 310027)
}

\section{Construction of a New Class of Oxindole-Based Axially Chiral Styrenes via Kinetic Resolution}

\author{
Jiang, Mengxue ${ }^{a} \quad$ Zhou, Tao $^{*, b}$ Shi, Bingfeng ${ }^{*, b}$ \\ ( ${ }^{a}$ School of Biotechnology and Health Sciences, Wuyi University, Jiangmen, Guangdong 529020) \\ ( ${ }^{b}$ Department of Chemistry, Zhejiang University, Hangzhou 310027)
}

基于芳环的轴手性骨架广泛存在于天然产物和药 物分子中，也是很多常见的优势手性配体和催化剂的核 心结构，同时，其在材料化学中的应用也越来越广泛. 近年来, 轴手性化合物的发展特别迅猛, 但大部分研究 集中在联芳基轴手性化合物和杂芳环轴手性化合物 ${ }^{[1-2]}$. 相比较而言, 苯乙烯类轴手性化合物或者芳基烯烃轴手 性骨架的合成和应用发展却非常缓慢, 可能是由于其旋 转能垒较低、构型稳定性较弱. 最近几年, 一些催化不 对称构建轴手性苯乙烯或者芳基烯烃轴手性骨架的方 法才相继被报道. 谭斌课题组 ${ }^{[3]}$ 和间海龙课题组 ${ }^{[4]}$ 分别 报道了通过有机小分子催化的炔烃衍生物的不对称加 成反应合成苯乙烯轴手性化合物. 2016 年, 顾振华课题 组 ${ }^{[5]}$ 利用 Pd 催化的芳基溴化物和重氮化合物的交叉偶 联成功合成了轴手性二氢荎骨架. 2017 年, Smith 课题 组 ${ }^{[6]}$ 通过手性相转移催化剂催化的烷基化也实现了二氢 荎结构的轴手性分子的高效构建. 最近, 史炳锋课题 组 ${ }^{[7-8]}$ 分别利用瞬态手性辅基和 $\operatorname{Pd}(\mathrm{II}) /$ 焦谷氨酸 ( $L-p \mathrm{Glu}-$ $\mathrm{OH})$ 不对称碳氢键活化策略, 实现了一系列开链轴手性 苯乙烯化合物的构建. 然而, 此类轴手性化合物的骨架 类型和催化不对称合成方法极为有限, 因此, 发展新的 催化不对称方法, 用于此类轴手性苯乙烯/烯烃-芳烃骨 架的高效不对称合成具有重要的意义.

氧化吲哚是许多天然生物碱和药物分子的核心骨 架, 基于这一类骨架的衍生物受到化学家们的广泛关 注, 但是, 氧化吲哚的轴手性骨架却很少被报道. 2016 年, 江苏师范大学化学与材料科学学院石枫课题组 ${ }^{[9]}$ 发
现在布朗斯特酸(B-H)催化下, 靛红和邻氨基芐基吲哚 反应可以以很高的产率和 $Z / E$ 选择性生成一种新型的基 于氧化吲哚的轴手性苯乙烯骨架(Scheme 1a). 但是作者 经过大量的尝试发现, 手性布朗斯特酸 $\left(\mathrm{B}^{*}-\mathrm{H}\right)$ 作为催化 剂并不能控制该反应的立体选择性，只能得到外消旋的 轴手性苯乙烯化合物. 动力学拆分作为一种常见的有效 获得光学纯化合物的策略引起了作者的注意, 作者设想 通过对外消旋体 $\mathbf{3}$ 的催化动力学拆分, 也许能实现该类 轴手性骨架的不对称合成. 该策略面临的挑战是找到合 适的拆分试剂与消旋的产物 3 发生反应，同时能找到与 这个反应匹配的手性催化剂, 在催化轴手性苯乙烯的两 种阻旋异构体参与反应时, 能够产生足够的速率差异实 现拆分. 基于这个设计思路, 最近, 石枫课题组 ${ }^{[10]}$ 成功 实现了手性磷酸催化下基于氧化吲哚的苯乙烯化合物 与浐内酯的动力学拆分反应 (Scheme 1b). 经过大量的 条件篮选，作者发现该反应在 $(R)-3,3^{\prime}$-双(2-菜基)-1, $1^{\prime}$-联 菜酚膦酸酯作为催化剂, $\mathrm{R}^{3}$ 和 $\mathrm{R}^{4}$ 分别为 4 -氯苯基和苯基 的㗒内酯作为开环试剂时，能以高对映选择性获得基于 氧化吲哚的轴手性苯乙烯化合物 $(R)-3 \quad(74 \% \sim 98 \% e e)$, 同时也能以很好的立体选择性合成兼具轴手性与中心 手性的二酰胺开环产物 $(S a, S)-5$ (84:16 94:6 $d r$, $76 \% \sim 95 \% e e)$. 控制实验表明该反应可能的活化模型 如 Scheme 2 所示. 手性磷酸催化剂能通过氢键同时与 底物 3 及呋内酯相互作用, 从而活化苯乙烯上的氨基, 促使其进攻浐内酯发生开环反应，而与手性磷酸构型匹 配的阻旋异构体 $(S)$-3 与吅内酯的反应速率远远大于另

* Corresponding authors. E-mail: skybasket@126.com; bfshi@zju.edu.cn. Published online December 4, 2020. 
(a) Discovery of a new class of oxindole-based axially chiral styrene derivatives

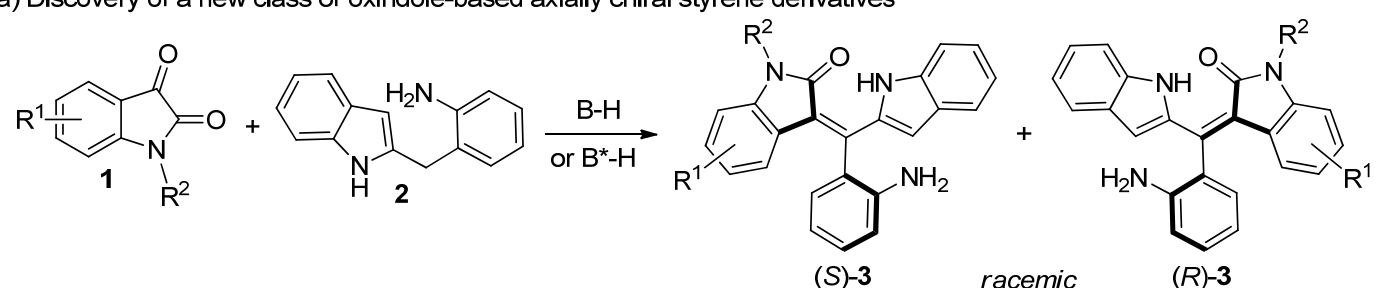

(b) Design of the catalytic $K R$ to access oxindole-based axially chiral styrenes<smiles>[R]c1ccc2c(c1)N([R])C(=O)C2=C(c1cc2ccccc2[nH]1)c1ccccc1N</smiles>

rac-3

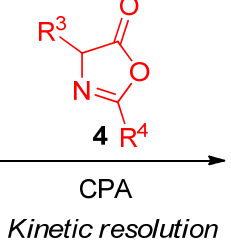

$S$ up to 106

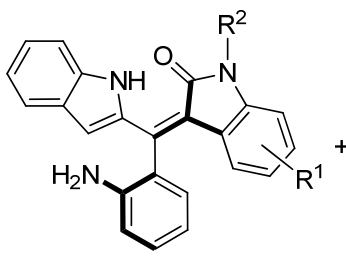

$(R)-3$

up to $98 \%$ ee

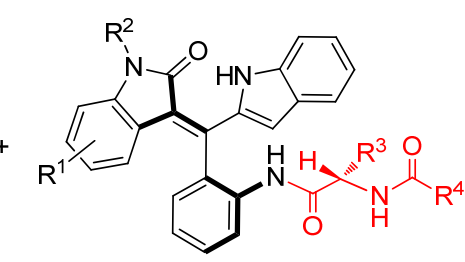

$\left(S_{a}, S\right)-5$

up to $94: 6 d r, 95 \%$ ee

图式 1 氧化吲哚类轴手性苯乙烯骨架的发现和动力学拆分

Scheme 1 Discovery and kinetic resolution (KR) of oxindole-based axially chiral styrene skeletons

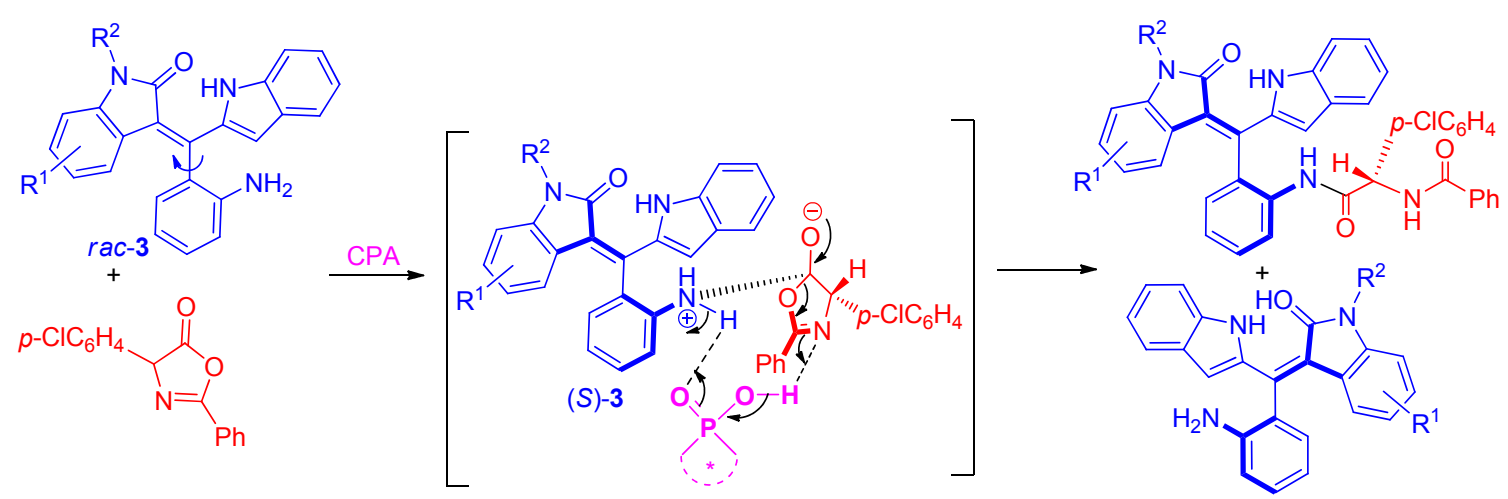

图式 2 可能的动力学拆分反应模型

Scheme 2 Plausible model of this KR reaction

一种阻旋异构体, 从而得到光学纯的反应物和开环产 物.

为了证明这一类新型骨架的潜在应用价值, 作者对 该反应进行了克级制备, 反应结果与 $0.1 \mathrm{mmol}$ 规模的 反应几乎相同. 同时作者对拆分的产物进行了多官能团 衍生化, 也对衍生物的应用进行了初步探索. 拆分出来 的产物 $(R)-3 \mathbf{a}$ 可以转化成新型的手性硫脲一叔胺类催化 剂 6, 手性硫艮衍生物 7 以及硫脲一叔膦衍生物 8 和 $\mathbf{1 0}$, 硫脲一叔膦衍生物可以进一步转化成硫脲一硫化膦衍生 物 9 和 11, 转化过程中对映选择性都能够保持(Scheme 3). 手性硫脲一叔胺 6 可以高效地催化邻亚甲基苯醌 12 与丙二腈 13 的不对称 $[4+2]$ 环化反应, 催化效果大大优 于常用的手性硫嫝一叔胺类催化剂(Scheme 3a). 轴手性 硫脲一叔膦衍生物 10 作为手性有机小分子催化剂能够催 化联烯 15 与靛红的烯烃衍生物 16 发生 $[4+2]$ 环加成反 应(Scheme 3b), 都能获得了良好的收率、优秀的非对映 选择性, 并且能够在一定程度上控制反应的对映选择 性. 这些结果表明, 此类基于氧化吲哚的轴手性苯乙烯
骨架很有希望开发成新型的轴手性有机小分子催化剂.
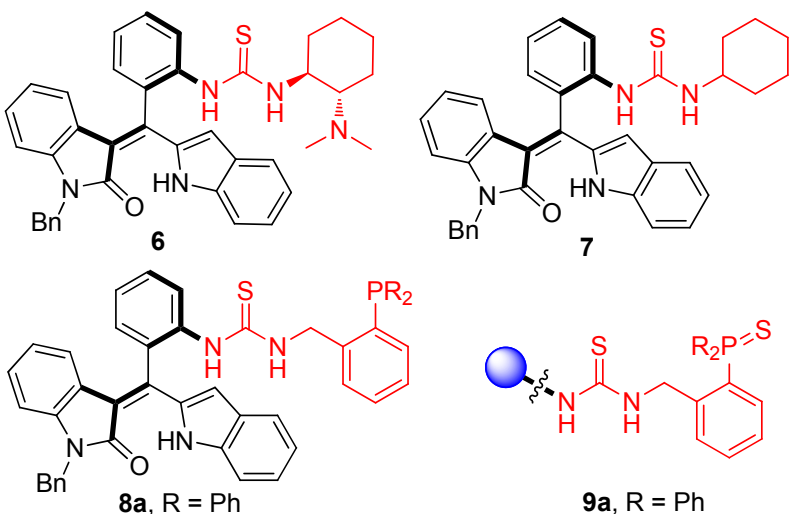

$8 b, R=C y$

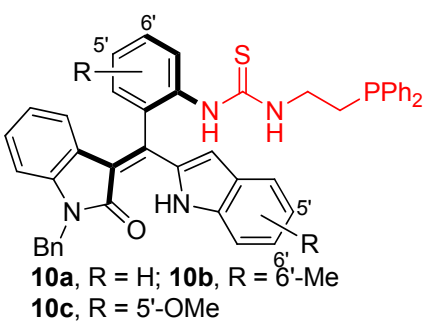

9a, $\mathrm{R}=\mathrm{Ph}$

9b, R = Cy

$O=$ axially chiral scaffold Cy = cyclohexyl<smiles>O=C(NC(=S)NCCP(=S)(c1ccccc1)c1ccccc1)c1ccccc1</smiles>

11 
(a)<smiles>NPC=C1C=C2OCOC2=CC1=O</smiles>

12 13 $\mathrm{PMP}=p-\mathrm{MeOC}_{6} \mathrm{H}_{4}$

\section{$\sum_{\mathrm{CN}}^{\mathrm{CN}} \underset{\text { toluene }}{\mathbf{6 ( 6 \mathrm { mol } \% )}}$} $-50^{\circ} \mathrm{C}, 4 \mathrm{~h}$ 13<smiles>C=C=C(CC(=O)OCC)C(=O)OCC</smiles>

15<smiles>[R]N1C(=O)C(=C(C#N)C#N)c2ccccc21</smiles>
$16^{R}$<smiles>N#CC1=C(N)Oc2cc3c(cc2[C@@H]1P(N)[Na])OCO3</smiles>

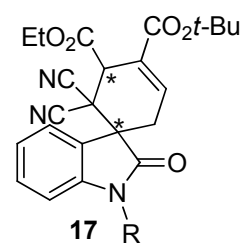
$>95: 5 d r, 32 \% \sim 41 \%$ ee
$90 \%$ yield, $91 \%$ ee

$63 \% \sim 91 \%$ yield

$\mathrm{R}=\mathrm{Bn}, \mathrm{Ph}, 1-$ naphthylCH $\mathrm{CH}_{2}$, 2-naphthylC $\mathrm{CH}_{2}$

图式 3 氧化吲哚的轴手性苯乙烯的应用

Scheme 3 Applications of oxindole-based axially chiral styrenes

综上所述, 江苏师范大学石枫课题组首次以动力学 拆分的策略实现了新型的基于氧化吲哚的轴手性苯乙 烯化合物的合成. 该策略为高对映选择性合成轴手性苯 乙烯类化合物的合成提供了一种新的思路，同时丰富了
轴手性苯乙烯化合物的类型. 作者也对这一类的轴手性 苯乙烯骨架的应用做了探索，证明了其在催化不对称合 成中潜在的应用价值.

\section{References}

[1] Wang, Y.-B.; Tan, B. Acc. Chem. Res. 2018, 51, 534.

[2] Zhang, S.; Liao, G.; Shi, B.-F. Chin. J. Org. Chem. 2019, 39, 1522 (in Chinese). (张硕，廖港，史炳锋，有机化学, 2019, 39, 1522.)

[3] Zheng, S.-C.; Wu, S.; Zhou, Q.; Chung, L.W.; Ye, L.; Tan, B. Nat Commun. 2017, 8, 15238.

[4] Jia, S.; Chen, Z.; Zhang, N.; Tan, Y.; Liu, Y.; Deng, J.; Yan, H. J. Am. Chem. Soc. 2018, 140, 7056

[5] Feng, J.; Li, B.; He, Y.; Gu, Z. Angew. Chem., Int. Ed. 2016, 55, 2186.

[6] Jolliffe, J. D.; Armstrong, R. J.; Smith, M. D. Nat. Chem. 2017, 9 , 558.

[7] Song, H.; Li, Y.; Yao, Q.-J.; Jin, L.; Liu, L.; Liu, Y.-H.; Shi, B.-F. Angew. Chem., Int. Ed. 2020, 59, 6576.

[8] Jin, L.; Yao, Q.-J.; Xie, P.-P.; Li, Y.; Zhan, B.-B.; Han, Y.-Q.; Hong, X.; Shi, B.-F. Chem 2020, 6, 497.

[9] Liang, J.; Zhang, H.-H.; Wang, C.-S.; Wu, Q.; Shi, F. Synthesis 2016, 48, 4548.

[10] Ma, C.; Sheng, F.-T.; Wang, H.-Q.; Deng, S.; Zhang, Y.-C.; Jiao, Y.; Tan, W.; Shi, F. J. Am. Chem. Soc. 2020, 142, 15686.

(Cheng, F.) 